\title{
EL CÓNCLAVE DE 1769 EN LA CORRESPONDENCIA DIPLOMÁTICA
}

\author{
Francisco José BELMONTE MAS
}

Universidad de Alicante

\begin{abstract}
Resumen
El mismo día de la muerte de Clemente XIII, el 3 de febrero de 1769, los embajadores de España, Francia y Nápoles en Roma se pusieron a trabajar con ahínco en la elección de un pontífice proclive a los intereses comunes de las tres coronas borbónicas. El objetivo principal del ministro de Carlos III, era promover a un candidato que previamente se comprometiese a extinguir la orden. Después de casi cinco meses de deliberaciones, entrevistas y negociaciones secretas, y la caída de diversos candidatos propuestos por cada una de las distintas facciones, Ganganelli resultó elegido de modo consensuado, aunque la documentación no permite afirmar la existencia de pacto simoníaco.
\end{abstract}

\begin{abstract}
The 3rd of April of 1769, the very same day Clemente XIII died, the ambassadors of Spain, France and Naples at Rome began to work hard on the election of a pontiff inclined to the common interests of the three borbon monarchies. To promote a candidate previously determined to extinguish the order was the main objective of the minister of Charles III. Deliberations, interviews and secret negotiations led to the raise and fall of the successive candidates proposed by the different parties. After nearly five months, Ganganelli was elected, even though the documentation is not accurate enough to assert the existence of «simoniac pact».
\end{abstract}

El día 3 de febrero de 1769 el embajador de España en Roma, Tomás Azpuru, enviaba una escueta carta a Grimaldi, Secretario de Estado, dándole cuenta de la muerte de Clemente XIII: «Ayer jueves por la noche, a las cuatro horas de este reloj, asaltó al Papa un accidente que en poco tiempo le quitó la vida...»'. La súbita muerte de Clemente XIII, a pesar de estar frecuentemente aquejado de dolencias y alejado de los asuntos vaticanos, cogió totalmente desprevenidos a los embajadores borbónicos

1. A.G.S., Estado, leg. 5012, Azpuru a Grimaldi, Roma, 3 de febrero de 1769. 
y a sus gobiernos. Azara, agente de preces del reino de España en Roma, plasmaba así la situación en que habían quedado: «...ha llegado el caso que deciamos ser necesario tener previsto y preparado: y ni uno ni otro. Estos señores ministros llos embajadores borbónicos] están sin luz y sin instrucción, como habrán escrito a vds. y esperan que les ilumine el Espíritu Santo..., hagan vds. lo que quieran, yo estoy persuadido a que en este cónclave hemos de hacer la figura más ruin que hemos hecho jamás...» ${ }^{2}$. Esta opinión quedó corroborada cuando llegó a España la noticia del fallecimiento del papa el día 21 de febrero, el propio Secretario de Estado español, Grimaldi, reconocía a su embajador en Roma cómo esta muerte les cogía por sorpresa, pudiéndose entreleer en la misma carta la falta de previsión del gobierno de Madrid: «En esta estrechez de tiempo apenas le ha habido para pensar en disposiciones algunas» ${ }^{3}$.

Mientras se esperaban órdenes concretas de las cortes borbónicas, la primera medida tomada por los embajadores de la casa de Borbón fue la de celebrar una reunión, el día 3 de febrero de madrugada, en el real palacio de España. A esta embajada española acudieron el embajador de Francia, marqués D'Aubeterre, y el de Nápoles, cardenal Orsini, siendo su anfitrión el embajador español, monseñor Azpuru. Pronto se pusieron de acuerdo en la necesidad de mantenerse unidos «de modo que sea una sola voz la de los tres y uno el espíritu que nos aliente... ${ }^{4}$. Al mismo tiempo decidieron seguir las pautas marcadas en el viejo plan de 21 de enero de 1766, remitido por el gobierno de España a Azpuru, para caso de cónclave imprevisto. Tanto Orsini como D'Aubeterre fueron hechos partícipes de los aspectos tratados en dicha instrucción. La principal cuestión sobre la que se pusieron de acuerdo fue la de abandonar la Ciudad Eterna en caso de realizarse una elección papal por parte de los cardenales de la curia sin esperar a sus colegas extranjeros. Era imprescindible advertir de esta posibilidad al colegio de cardenales y por ello se acordó visitar a los purpurados antes de su entrada en cónclave y «manifestarles que Sus Magestades [Carlos III, Luis XV y Fernando IV] no podrán oir con indiferencia una precipitada elección sin dar tiempo a que puedan concurrir los vocales forasteros... $\gg^{5}$. Había que evitar a toda costa que el «partido de los jesuitas», con amplio respaldo en el Sacro colegio, elevara al solio pontificio a alguno de sus parciales. $Y$ en este sentido los tres ministros coincidieron en la exclusión de cuatro cardenales: «a quattro exclusi dalle tre corti... Castelli, Braschi, Buonacorsi e Torrigiani...» ${ }^{6}$. Pese a todas las precauciones, los embajadores borbónicos mostraban su impotencia por boca de D'Aubeterre a la hora de tener la certeza de que se decantaban por un cardenal alejado del mundo jesuítico, lo que da-

2. Azara a Roda, Roma, 9 de febrero de 1769, El espiritu de D. José Nicolás de Azara descubierto en su correspondencia epistolar con D.Manuel de Roda, Madrid, 1846, t. I, pps. 212-213.

3. A.G.S., Estado, leg. 5012, Grimaldi a Azpuru, El Pardo, 21 de febrero de 1769.

4. A.M.AA.EE., Santa Sede, leg. 332, Azpuru a Grimaldi, Roma, 6 de febrero de 1769.

5. Ibídem.

6. A.G.S., Estado, leg. 5012, Orsini a Grimaldi, Roma, 6 de febrero de 1769. 
ba idea de la intrincada red de alianzas y apoyos que la Orden de San Ignacio había tejido en la capital del catolicismo: «Ce n'est pas que nous croyons plus assûrés des autres: au moyen des affiliations secretes, il est impossible de pouvoir juger de leurs vrais sentiments pour la Societé, au point que si par hypotese on s'en remettait à moi pour le choix d'un Pape, j'y serais tellement embarasse, que je ne connaitrais d'autres moyens pour le faire que celui du sort. Pendant le dernier pontificat qui a duré dix ans et demy, les jésuites ont été les maitres de toutes les graces, d'où on peut juger de la quantité de créatures qu'ils se sont faites qui ont eu grande de se cacher et qu'il n'y a pas moyens de distinguer» '.

El día 15 de febrero «a medianoche» ${ }^{8}$ se reunieron en cónclave los cardenales residentes en Roma. El Sacro colegio, que debía estar compuesto por 70 vocales, contaba con 13 vacantes; es decir, el número de cardenales quedaba reducido a 57 . No obstante esa cifra, después de muchas semanas, e incluso meses, el cónclave quedó definitivamente constituido cuando concurrieron dos de los tres cardenales españoles, siendo el número final de purpurados asistentes al cónclave 46 . Los achaques y el peso de los años frustraron, en algunos casos, su asistencia al cónclave. De cualquier modo en el primer escrutinio celebrado el día 16 de febrero sólo se hallaban presentes en la Capilla Sixtina 28 cardenales. Y al parecer 16 de ellos estaban en la órbita jesuítica". El peligro de una elección sorpresiva parecía inminente: el partido projesuítico estaba rozando, si es que no contaba ya con ella, temía Azpuru, la mayoría de los dos tercios necesaria para aupar a su candidato a la barquilla de san Pedro. Dado el escaso margen con que contaban y la situación tan precaria en la que se encontraba el partido de los cardenales afectos a las cortes, se llegó a conminar con duras reconvenciones a todo aquel cardenal susceptible de cambiar su opinión. D'Aubeterre, a juzgar por el «plan» que envió a Choiseul, debió mantener una conversación muy tensa con uno de estos cardenales: «Je dois aussi parler au cardinal Lante... d'ailleurs dans le dernier conclave il se conduisit très mal. Je compte lui dire très nettement que s'il continuait de se conduire de meme dans celui cy, le Roy ne regarderait plus la Maison comme lui étant attachée et retirerait la protection qu'il lui accorde: que de plus j'ignore ce qu'il arriverait peut être des reventus qu'il possede en France: ce dernier moyen ne lui ser pas insensible, étant d'une avarice sordide. D'ailleurs je ne connais point d'autre façon de pouvoir s'en assûrer. J'espere que le Roy ne désaprouvera pas que pour le bien de son service je parle ainsi à ce cardinal.» ${ }^{10}$

Una advertencia similar debió recibir el cardenal napolitano Pirelli; Azpuru dudaba de su fidelidad hacia el partido de los coronas y sospechaba haría causa común con el partido Rezzonico. Por ello advertía a Orsini: «Su Eminencia sabrá muy bien

7. A.G.S., Estado, leg. 5012, D'Aubeterre a Choiseul, Roma, 6 de febrero de 1769.

8. PASTOR, L.: Historia de los Papas en la época de la monarquía absoluta, Vol. XXXVI, Clemente XIV, (1769-1774), Barcelona, 1937, p. 10.

9. A.G.S., Estado, leg. 5012, Azpuru a Grimaldi, Roma, 25 de febrero de 1769.

10. A.G.S., Estado, leg. 5012, D'Aubeterre a Choiseul, Roma, 6 de febrero de 1769. 
cómo lo ha de tratar, y cuándo convendrá el insinuarle que la persona en quien pone la mira el partido Rezzonico no puede ser del gusto de su Soberano, el rey de las Dos Sicilias.» ${ }^{11}$. El fin de todas estas advertencias era conseguir un partido capaz de dar, por sí solo, la exclusiva de votos. ${ }^{12}$ Logrado ese objetivo prioritario se podría, por un lado, impedir una elección precipitada o una elección de un cardenal no grato a las cortes $y$, por otro, dado que ningún bando contaba con la mayoría de los dos tercios de los votos, negociar algunas condiciones bajo las cuales subiría algún purpurado al solio pontificio. En principio, el número de cardenales amigos de la casa de Borbón rondaría la cifra necesaria para lograr esa exclusiva de votos. Azpuru tenía serias dudas de alguno de esos purpurados, mientras que Orsini y D'Aubeterre consideraban haber alcanzado el número suficiente: «Me persuado que tenemos cuantos cardenales bastan para la exclusiva, aunque no hago las cuentas tan alegres como el embajador de Francia y Orsini, pues aquél la forma de los dos Corsinis, Yorck, Ganganelli, Guglielmi, Negroni, Lante, Perrelli, Stoppani, Pirelli y Orsini; y éste se extiende hasta doce, que avisó ayer por la noche desde el cónclave tener seguros para la exclusiva, pero yo dudo de alguno, y me contento con diez, que bastan en el presente estado para dicha exclusiva. ${ }^{13}$

De los tres embajadores borbónicos ante el Vaticano sólo uno de ellos, Orsini, era cardenal. Por tanto, desde el día 15 de febrero, y en tanto no llegaran a Roma los cardenales franceses y españoles, la única voz de una corte borbónica dentro del cónclave era la suya. En la primera reunión de los tres embajadores, al día siguiente de la muerte de Clemente XIII, ya se acordó de manera implícita que Orsini sería el encargado de negociar ante el Sacro colegio representando a las tres coronas. Faltaba la autorización expresa de las cortes. Grimaldi a finales de febrero remitía dicha autorización de la corte de España para el cardenal napolitano: «como interim lleguen los cardenales españoles es necesario haya en el cónclave quien pueda poner en práctica lo que ocurra en nombre del rey, ha resuelto S.M. dar a V.Ema. interinamente este encargo... y encarga el rey a V.Ema. no sólo que le avise [a Azpuru] de todo [lo que ocurre en el cónclavel con exactitud, sino que ejecute lo que en nombre de S.M. le previniese según la ocurrencia de las cosas. ${ }^{14}$. Sin embargo, la relación entre los representantes españoles en Roma, Azpuru y Azara, y los napolitanos, Orsini y su superior en Nápoles, Tanucci, no parecía ser muy buena. Tanucci hablaba así a Orsini de los españoles: «cotesti due spagnoli malignano tutto, e non sono amici ne di V.E. ni mici; e barbaro essendo, sono nemici di tutto non spagnolo, e particolarmente degl' $i$ taliani.» ${ }^{15}$ Por su parte Azpuru siempre mantuvo su recelo respecto a la figura del cardenal napolitano; tampoco Azara sentía mucha simpatía hacia éste. Antes de la en-

11. A.G.S., Estado, leg. 5012, Azpuru a Grimaldi, Roma, 25 de febrero de 1769.

12. Dar la exclusión o exclusiva significaba no dar el voto a un candidato o bien no incluir el nombre de un cardenal en la lista de los papables.

13. A.G.S., Estado, leg. 5012, Azpuru a Grimaldi, Roma, 16 de febrero de 1769.

14. A.G.S., Estado, leg. 5013, Grimaldi a Orsini, El Pardo, 28 de febrero de 1769.

15. A.R.S.I., Hist. Soc., lib. 257, Tanucci a Orsini, Venafro, 28 de febrero de 1769 
trada del Sacro colegio en la Capilla Sixtina, el 9 de febrero, ya advertía a Roda que sería Orsini el coordinador de la política de las tres coronas dentro del cónclave en tanto no llegasen los cardenales nacionales: «Todo nuestro andamio estriba sobre la gran cabeza de Orsini, y de Dionigi, su conclavista, más jesuita que el Padre Ricci, y más tonto que su amo.» ${ }^{16}$

A pesar de todo, hasta la llegada de los dos cardenales españoles, a finales de abril, Orsini no fue relevado de su puesto y por sus puntuales billetes, donde se incluían los escrutinios de cada día, tanto Azpuru como Grimaldi recibían información sobre la marcha del cónclave.

Una de las principales preocupaciones de los ministros de las cortes borbónicas fue la de conseguir situar a cada uno de los cardenales en las distintas facciones, o partidos, que tenían cabida dentro del cónclave. Para los citados ministros el Sacro colegio se dividía en tres partidos principales. La facción más poderosa era la comandada por el cardenal Rezzonico. En el seno de este partido de cardenales curiales, conocedores del funcionamiento y de todos los entresijos del Vaticano, tenían cabida diferentes tendencias, aunque el denominador y causa común de todos ellos era la defensa de las inmunidades de la Iglesia y, estrechamente ligado a ello, el apoyo a la Compañía de Jesús.

Un segundo partido estaba formado por los llamados cardenales «indiferentes», compuesto por un número de vocales reducido pero que no se podía desdeñar; de él podría depender el ascenso o la caída de los candidatos a ceñirse la tiara papal.

Por último, el tercer grupo en liza era el partido de los coronas o nacionales, poco numeroso en principio pero con mucha voz dentro del cónclave. A esta tercera facción no pertenecían, en sentido estricto, todos los purpurados foráneos, también había curiales; asimismo, concurrían aquellos cardenales poco amigos de la Compañía de Jesús o bien discriminados durante el pontificado del papa Rezzonico. Algunos cardenales se vieron obligados, so pena de caer en desgracia y perder sus rentas, a tomar parte de él.

Además de tratar de situar a los cardenales en cada uno de los principales partidos, los ministros borbónicos elaboraron una serie de listas donde se clasificaba a los cardenales, catalogándolos genéricamente como «buenos, malos o dudosos». Una de estas primeras listas dividía a los purpurados en tres categorías:

«Seguros: Cavalchini, Lante, Yorck, Stoppani, Malvezzi, Sersale, Solís, Luynes, Guglielmi, Conti, La Cerda, Branciforte, Pirelli, Neri Corsini, Orsini, Bernis, Caracciolo, Perrelli, Andrés Corsini, Canale.

Dudosos: Pozzobonelli, Ganganelli, Colonna, Pallavicini, Pamfili, Negroni.

Contrarios: Juan Francisco Albani, Serbelloni, Lanze, Rezzonico, Prioli, Rossi, Spínola, Castelli, Fantuzzi, Molino, Buonacorsi, Bufalini, Boschi, Calino, Borromei, Parraciani, Alejandro Albani, Chigui, Torrigiani, Veterani.» ${ }^{17}$

16. Azara a Roda, Roma, 9 de febrero de 1769, El espíritu..., t. I, p. 213.

17. A.G.S., Estado, leg. 5013. 
El número de cardenales súbditos del rey español, Carlos III, se reducía tan sólo a tres: Luis Antonio Fernández de Córdova, arzobispo de Toledo; Francisco de Solis Folch de Cardona, arzobispo de Sevilla, y Buenaventura Spínola de la Cerda, arzobispo de Neocesárea y Patriarca de las Indias Occidentales. A pesar de ser meramente un número casi testimonial, su participación en la elección papal de 1769, dada la importancia de este cónclave y el escaso margen con que contaba el partido de las coronas, era inexcusable. El único que no iba a partir hacia la Ciudad Eterna era el arzobispo de Toledo: «Inmediatamente hice sabidor de la voluntad del rey al cardenal Patriarca y despaché un extraordinario al cardenal de Solis; y si se omitió ejecutar lo mismo con V. Ema., no fue porque S.M. no se halle íntimamente persuadido de que en V. Ema. concurren los mismos deseos que en S.M.... sino por considerarle imposibilitado de transferirse a Roma.» ${ }^{18}$ No se puede dudar de las buenas intenciones que movían al gobierno de Madrid a la hora de prescindir del arzobispo de Toledo; aunque, probablemente, en esa meditada decisión se tuvo en cuenta el alineamiento, tan sólo dos años antes, del de Toledo, con los jesuitas españoles con motivo de su expulsión. ${ }^{19}$ De los dos cardenales restantes, en la propia corte de Madrid, no parecía tenerse muy buen concepto: «todos ellos de la primer grandeza, pero de escaso talento y poca doctrina.» ${ }^{20}$ A pesar de esta consideración, desde el gobierno de Madrid se decidió su rápida partida hacia Roma. Finalmente, después de dos meses de infructuosas tentativas llegaba a la Ciudad del Tíber el primero de los cardenales españoles y, presuroso, escribía al Secretario de Estado español: «Antes de ayer [25 de abril] llegué a ésta, y ayer por la mañana dí cuenta al Sacro colegio de mi arribo, por lo que se ha dispuesto que esta noche me encierre en el cónclave, sin esperar al cardenal de Solís, que aún no sé positivamente cuando llegará, aunque se dice que será en breve.» ${ }^{21}$

El último día del mes de abril el segundo de los cardenales españoles se reunía en la Capilla de Sixto con el resto de los purpurados, quedando definitivamente constituido el colegio cardenalicio dentro del cónclave en 46 vocales. Los mismos que, 19 días más tarde, elevarían al solio pontificial al sucesor de Clemente XIII. ${ }^{22}$

En el mes de febrero, en espera de órdenes concretas de las cortes, los embajadores borbónicos, salvo Orsini, eran partidarios de solicitar al cónclave la extinción de la Compañía de Jesús. La corte de Madrid era donde más seguidores tenía esta propuesta aunque, en principio, también se adhirió el jefe del gobierno napolitano, Tanucci, si bien después cambió de opinión. El embajador de Francia mostró, asimismo su absoluta disposición para formular al cónclave dicha solicitud, enfrentándose, incluso, a los cardenales franceses y al parecer de su Secretario de Estado, Choiseul. La postura de España parecía clara, todas las negociaciones debían tener como eje

18. A.G.S., Estado, leg. 5013, Grimaldi al cardenal arzobispo de Toledo, El Pardo, 28 de febrero de 1769.

19. PINEDO, I.: Manuel de Roda. Su pensamiento regalista, Zaragoza, 1983, pps. 159-160.

20. A.G.S., Estado, leg. 6102, Grimaldi a Tanucci, El Pardo, 21 de febrero de 1769.

21. A.G.S., Estado, leg. 5013, Solis a Grimaldi, Roma, 30 de abril de 1769.

22. A.G.S., Estado, leg. 5013, Solis a Grimaldi, Roma, 30 de abril de 1769. 
central la extinción de la Compañía de Jesús: «Quiere S.M. [Carlos III] que se trate y hable solamente de la extinción de la Compañía.» ${ }^{23}$

Orsini, previa deliberación con los cardenales franceses se desmarcó de la propuesta; argumentaban que el Sacro colegio ni tenía autoridad, ni estaba facultado para tratar semejante solicitud y advertían del peligro que podría acarrear su planteamiento para los intereses de la liga borbónica. ${ }^{24}$ Azpuru, a pesar de las persistentes reticencias y negativas dadas por los cardenales franceses y Orsini, contaba con el apoyo de D'Aubeterre. Sin embargo, a finales de abril, desde París se ordenó a su embajador en Roma no insistir más en el asunto; se consumaba la defección de Francia y Azpuru quedaba solo en sus planteamientos: «D'Aubeterre me dijo que presentemente en el cónclave no se podía tratar de las condiciones que se han propuesto para el acomodamiento con estas cortes, pues vemos que aún la de extinción de jesuitas tiene la contradicción que experimentamos, y que a nuevo papa, se podrá entablar este tratado y proponer dichas condiciones, y se deberá esperar, como dice dicho embajador, a la elección del nuevo papa... ${ }^{25}$

La entrada en el cónclave de Solís y De la Cerda puso fin a más de dos meses de tensa inactividad, durante los cuales no despuntó significativamente ningún candidato en los más de 150 escrutinios realizados. En las entrevistas previas a su encierro, Azpuru instruyó a ambos purpurados de la marcha de la negociación así como de las instrucciones recibidas desde la corte de España. El embajador español había agotado todos sus argumentos frente a las constantes negativas de los cardenales franceses y Orsini para proponer a algún candidato que aceptara el compromiso de una futura extinción canónica del Instituto de san Ignacio. Los cardenales españoles serían la última vía empleada por Azpuru en su empeño. Así se lo hacía saber a Solís: «quedamos de acuerdo en que V. Ema. manifieste a los cardenales franceses y Orsini su firmeza en no dar su voto, ni consentir en la elección de quien no hiciese dicha promesa, sino por escrito, a lo menos verbal, ante los cardenales que lleven la voz por las cortes de la casa de Borbón, para poderle reconvenir con la palabra que ahora diere cuando hecho papa se excuse, o niegue a la extinción de la Compañía.» ${ }^{26}$

Solís transmitió estas ideas en el primer encuentro mantenido con el resto de cardenales borbónicos dentro del cónclave, celebrado el 3 de mayo en la celda de Orsini. Pero los franceses argumentaron en contra de dicha propuesta que aún en el caso de obtener un escrito firmado por el candidato a la tiara papal, éste, una vez elegido papa, podría declararlo carente de validez, objetando la nulidad misma del documento y amparándose en una reflexión más profunda y meditada. Llegados a este punto las cortes no podían presionar al Sumo Pontífice, porque el escándalo de una elección simoníaca conmovería a todo el mundo católico. Finalizaban su razonamiento expli-

23. A.M.AA.EE., Santa Sede, leg. 332, Azpuru a Grimaldi, Roma, 30 de marzo de 1769.

24. A.G.S., Estado, leg. 5013, Orsini a Azpuru, Dal conclave, 1 de abril de 1769.

25. A.M.AA.EE., Santa Sede, leg. 332, Azpuru a Grimaldi, Roma, 20 de abril de 1769.

26. A.G.S., Estado, leg. 5013, Azpuru a Solís, Roma, 2 de mayo de 1769. 
cando la necesidad de reunir dos tercios de los votos para validar la propuesta, supuesto totalmente improbable. La intervención de Orsini reforzó la postura de los franceses: «Habiendo adherido el cardenal Orsini a su opinión propusieron que sería utilísimo tanto por la seguridad de sus conciencias, como por los intereses de las coronas, que no se tocase ese punto a algún cardenal antes de la elección: que se eligiese un papa del que se tuviese una moral seguridad de que abrazará después de electo las propuestas de las coronas... de este dictamen nunca se separarian, no podrían hacer otra cosa en servicio de sus soberanos que no intervenir en la elección, en el caso, que con lo propuesto no se conformasen.» ${ }^{27}$

Esa misma jornada, y después de concluído el primer encuentro de los cardenales borbónicos, Orsini anunciaba al embajador español el inicio de los contactos de los principales partidos del cónclave para poner fin a la inactividad de los dos últimos meses y medio. La cabeza visible del partido Rezzonico, el sobrino del difunto pontífice, mantuvo un encuentro con Cavalchini en su celda, explorando las intenciones del octogenario purpurado, a sabiendas de sus buenas relaciones con los cardenales nacionales. El anciano cardenal comunicó a Rezzonico el parecer de las cortes, que se declaraban dispuestas a elegir a un candidato «sabio, prudente e imparcial» y no opuesto a ellas. ${ }^{28}$ Al día siguiente de este primer encuentro la liga borbónica, compuesta por Orsini, los cardenales franceses y los españoles, inició su preceptiva ronda de contactos: «Después de haber conferenciado privadamente, pasamos a la celda de Cavalchini, que postrado en la cama, se excusó de mezclarse en negociaciones, desde donde fuimos a la de Pozzobonelli, que quedó bien informado de las comunes ideas y de allí a la del nepote Rezzonico, el que se aseguró que las intenciones de las cortes, eran las de dejar al Sacro colegio en plena libertad de elegir papa, pero que parecía conveniente que antes se propusiese al sujeto, o sujetos, que tuviesen por dignos: respondió, político, afable, que lo consultaría y que respondería presto, y que esperaba la total satisfacción de las cortes. ${ }^{29}$

El primer paso efectivo para desbloquear la elección papal fue el acuerdo adoptado para ir proponiendo candidatos al Sacro colegio y someterlos al refrendo de éste. Rezzonico planteó la posibilidad de presentar al cónclave a los candidatos en orden a la antigüedad, es decir, atendiendo a la fecha en que habían recibido la muceta cardenalicia. ${ }^{30}$ Aunque, finalmente, se pactó «que para hacer las propuestas se guardase la alternativa según la última práctica, empezando la propuesta por un cardenal del colegio viejo o Benedictino siguiendo la de los del colegio nuevo o Clementino.» ${ }^{31}$

Siguiendo este criterio los cardenales borbónicos comenzaron a recabar apoyos para la presentación de Cavalchini. Éste en el cónclave de 1758 estuvo muy cerca de

27. A.G.S., Estado. leg. 5013, Solís a Azpuru, Del cónclave, 3 de mayo de 1769.

28. A.G.S., Estado, leg. 5013, Orsini a Azpuru, Dal conclave, 3 de mayo de 1769.

29. A.G.S., Estado, leg. 5013, De la Cerda a Grimaldi, Del cónclave, 4 de mayo de 1769.

30. Ibídem.

31. A.M.AA.EE., Santa Sede, leg. 332, Azpuru a Grimaldi, Roma, 18 de mayo de 1769. 
ser elegido Sumo pontífice, pero Francia la presentó la exclusiva formal por su actitud abiertamente hostil al galicanismo y su cercanía a los jesuitas. A lo largo del pontificado de Clemente XIII dio muestras, sin embargo, de estar más alineado con las cortes que con la curia papal. Al comenzar el año 1769 los ministros borbónicos, y sobre todo el embajador español, habían mudado su concepto sobre el purpurado. ${ }^{32}$ Gracias a los buenos oficios de España, Luis XV le retiró la exclusiva presentada contra él diez años atrás pudiendo optar de nuevo a la tiara papal. ${ }^{33} \mathrm{Si}$ triunfaba la candidatura del anciano cardenal el beneficio para las cortes sería considerable: Cavalchini en el solio pontificio, y.aún por corto que fuera su papado, extinguiría a la Compañía de Jesús y situaría en los 13 capelos vacantes a purpurados contrarios al jesuitismo y afectos a las cortes. En caso de no contar con apoyos suficientes para erigirse en Sumo pontífice, las cortes lo situaban dentro del nuevo organigrama gubernamental del próximo papa ${ }^{34}$ No obstante, la propuesta de dicho purpurado no terminaba de recabar los apoyos necesarios dentro del cónclave: «hay que tener en cuenta que se ha quedado suspendida la candidatura del cardenal Cavalchini, del que ya no se habla, aunque sigue teniendo apoyos...». ${ }^{35}$ Finalmente Solís relataba cómo había caído el cardenal decano: «Se procuró explorar sigilosamente el número de voces que tenía, y viendo que le repugnaban los otros dos partidos, asi por su avanzada edad, como por su quebrantada salud, halló por conveniente el partido regio no se hiciese formal la propuesta...». ${ }^{36}$

Al no conseguir la propuesta del cardenal Cavalchini los apoyos necesarios, según lo establecido con el cardenal Rezzonico, el turno pasaba a algún cardenal de este partido. Fantuzzi era, en principio, uno de los mejores situados en los escrutinios y contaba con muchos apoyos en el partido Clementino e, incluso, en el opuesto. Azara decía de este purpurado: «fue hecho cardenal en 1759 sin ninguna pretensión... profesa la doctrina de San Agustín, y por consiguiente es poco afecto a los jesuitas...»."77 Roda lo calificaba como religioso «de buena doctrina y muy justificado». ${ }^{38}$ Pese a las protestas de Orsini, que también apoyó a Fantuzzi, Azpuru consiguió hacer valer su opinión ante Grimaldi. Primeramente el cardenal Fantuzzi fue incluido en el listado de los considerados «malos» y tras las reiteradas peticiones del embajador español, Grimaldi le envió la exclusiva formal para ser usada según su arbitrio. ${ }^{39}$ El propio Fantuzzi, conociendo su recusación por las cortes borbónicas, se excluyó de los candidatos a la tiara papal; Azpuru había logrado eliminar definitivamente a Gaetano

32. A.G.S., Estado, leg. 5012, Azpuru a Grimaldi, Roma, 16 de febrero de 1769.

33. A.G.S., Estado, leg. 5012, Grimaldi a Azpuru, El Pardo, 21 de febrero de 1769.

34. A.M.AA.EE., Santa Sede, leg. 332, Azpuru a Grimaldi, Roma, 27 de abril de 1769.

35. A.G.S., Estado, leg. 5013, Orsini a Azpuru, Dal conclave, 10 de mayo de 1769.

36. A.G.S., Estado, leg. 5013, Solis a Azpuru, Del cónclave, 16 de mayo de 1769.

37. A.G.S., Estado, leg. 5012, Azara a Grimaldi, Roma, 5 de junio de 1766.

38. A.G.S., Estado, leg. 5012, Dictamen de Roda, 23 de febrero de 1769.

39. A.G.S., Estado, leg. 5012, Grimaldi a Azpuru, Aranjuez, 4 de abril de 1769. 
Fantuzzi. Un día antes de la elección papal resumía a Grimaldi la renuncia de este cardenal: «se determinó darle la exclusiva siempre que de otro modo no se pudiese destruir su partido, y habiendo llegado Fantuzzi a penetrarlo, y precaviendo su desaire, escribió un billete abierto al cardenal Rezzonico, pidiéndole que de ningún modo se le propusiese, pues aún en el caso de que se le quisiese elegir y ofreciese la tiara, renunciaría...». ${ }^{40}$

Descartado Fantuzzi las miras del partido Rezzonico se centraron en el cardenal Marcantonio Colonna. Desde la celebración de los primeros escrutinios hasta la finalización del éstos siempre contó con un respaldo importante. Respaldo considerado por Azpuru, continuamente preocupado por un posible golpe de mano del partido de los zelantes que al sumar los apoyos de Colonna y Fantuzzi en su verdadero candidato, Chigi ${ }^{41}$, lo elevaría al solio pontificio. A principios del mes de abril, el cardenal Colonna continuaba aglutinando apoyos lentamente. ${ }^{42}$ Pero no fue hasta el día 8 de mayo cuando su figura cobró verdadera importancia. ${ }^{43}$ Estudiada su candidatura por los cardenales borbónicos acordaron, en un primer momento, excluirla con los votos. ${ }^{44}$ En una segunda reunión los cardenales nacionales «empezaron a tomar medidas convenientes para que no se propusiese evitando el desaire... ${ }^{45}$ Los cardenales Bernis y De la Cerda fueron los encargados de transmitir a Rezzonico la resolución tomada por los componentes de la liga borbónica. Quedaban frustradas, de nuevo, las expectativas del partido celante; en esta segunda propuesta, a diferencia de Fantuzzi, no se argumentó que el candidato no era grato a las cortes: amparándose en su escasa edad, 45 años, la excusa fue idónea.

Las preferencias de la corte de España se centraron desde un primer momento en promocionar la candidatura de Sersale. Este cardenal, arzobispo de Nápoles y súbdito del rey de las Dos Sicilias, figuró en todas las listas elaboradas por las cortes de París y Madrid en la primera posición de los considerados «buenos» o «muy buenos». En Madrid no se tenía ninguna duda sobre la lealtad del arzobispo napolitano y se defendió abiertamente su candidatura. ${ }^{46}$ Los apoyos de las cortes a este cardenal no pasaron inadvertidos en el Sacro colegio y pronto se supo que era su candidato predilecto: «se ha esparcido la voz en el cónclave de que las cortes quieren la elección del cardenal Sersale...». ${ }^{47}$ Las excesivas alabanzas y el poco disimulado apoyo de las cortes hicie-

40. A.M.AA.EE., Santa Sede, leg. 332, Azpuru a Grimaldi, Roma, 18 de mayo de 1769.

41. El cardenal Chigi estaba fuertemente impregnado de los criterios de la curia romana y era, además, un significado aliado de la causa jesuítica. A.G.S., Estado, leg. 5012, Orsini a Grimaldi, Roma, 6 de febrero de 1769.

42. A.M.AA.EE., Santa Sede, leg. 332, Azpuru a Grimaldi, Roma, 6 de abril de 1769.

43. A.G.S., Estado, leg. 5013, Azpuru a Sebastián García, Real palacio de España, (Roma), 8 de mayo de 1769.

44. A.G.S., Estado, leg. 5013, Solís a Azpuru, Del cónclave, 10 de mayo de 1769.

45. A.G.S., Estado, leg. 5013, Solís a Azpuru, Del cónclave, 18 de mayo de 1769.

46. A.G.S., Estado, leg. 5012, Dictamen de Roda, 23 de febrero de 1769.

47. A.M.AA.EE., Estado, leg. 332, Azpuru a Grimaldi, Roma, 23 de febrero de 1769. 
ron crecer la desconfianza del colegio cardenalicio hacia el napolitano. Así Orsini advertía de sus escasas posibilidades: «por ser muchos los que se han declarado contrarios a dicho Emmo...». ${ }^{48}$ A medida que avanzaban las negociaciones para presentar los candidatos al Sacro colegio las escasas opciones de Sersale fueron desapareciendo; Solís informaba a Madrid del rechazo que los curiales demostraban por el arzobispo de Nápoles: «a quien los contrarios deshechan por realista». ${ }^{49}$ Las «fuertes tentativas» realizadas en favor de Sersale no dieron resultado alguno y ni siquiera fue propuesto como candidato.

Si Sersale fue el candidato más apreciado por España, Stoppani lo fue de Francia. En efecto, Francia le apoyó desde un principio y también cosechó un respaldo considerable del partido celante y del cardenal Orsini. No contó, sin embargo, con el visto bueno de Azpuru ni de la corte de Madrid. ${ }^{50}$ Terminado el mes de abril el reiterado apoyo de Francia a este purpurado daba sus frutos y Azpuru se vio obligado a transigir. El embajador español aceptaba incluirlo como «bueno», añadiendo una condición: «subsidiariamente y cuando no hubiere proporción para sacar nuestro partido por papa al cardenal Sersale o alguno de los otros que desde un principio se habian considerado buenos». ${ }^{51}$ Comenzado el mes de mayo las primeras informaciones de Solís a Azpuru hablaban de un letargo en la candidatura del citado cardenal: «parece que el cardenal Stoppani está sepultado en el olvido, pero se discurre saldrá a su tiempo». ${ }^{52}$ Las reiteradas negociaciones llevadas a cabo por los franceses para reforzar la candidatura de este cardenal acabaron volviéndose en su contra. Fue precisamente el excesivo empeño de la corte de Francia hacia este purpurado lo que hizo crecer las sospechas de los celantes hacia él, retirándole sus apoyos iniciales. Sin el beneplácito de los curiales ninguna candidatura podía prosperar y el mismo embajador de Francia se vio obligado a dejar en suspenso la propuesta de Stoppani. ${ }^{53}$

Con objeto de desbloquear la elección pontificia, el día 10 de mayo el cardenal arzobispo de Sevilla solicitaba a Azpuru permiso para entablar conversaciones con uno de los Albanis: «Solís, sin embargo de estar enterado del carácter del cardenal Juan Francisco Albani, y de las órdenes repetidas de su corte de que en la elección pontificia no se proceda sin el acuerdo de los cardenales franceses con Orsini, atentas las circunstancias presentes y visto que puede fundarse alguna otra esperanza en Juan Francisco Albani... juzga el cardenal Solís este paso por muy útil, sin participarlo al congreso regio... ${ }^{54}$ Azpuru respondía a Solís advirtiéndole del peligro que suponía para la liga borbónica iniciar por cuenta propia cualquier negociación con el

48. A.M.AA.EE., Santa Sede, leg. 332, Azpuru a Grimaldi, Roma, 23 de marzo de 1769.

49. A.G.S., Estado, leg. 5013, Solís a Azpuru, Del cónclave, 7, 8 y 9 de mayo de 1769.

50. A.M.AA.EE., Santa Sede, leg. 332, Azpuru a Grimaldi, Roma, 6 de abril de 1769.

51. A.M.AA.EE., Santa Sede, leg. 332, Azpuru a Grimaldi, Roma, 27 de abril de 1769.

52. A.G.S., Estado, leg. 5013, Solís a Azpuru, Del cónclave, 7, 8 y 9 de mayo de 1769.

53. A.G.S., Estado, leg. 5013, Azpuru a Sebastián García, Real palacio de España, 16 de mayo de 1769.

54. A.G.S., Estado, leg. 5013, Solís a Azpuru, Del cónclave, 10 de mayo de 1769. 
cardenal Albani: «La duda que tiene Su Ema. sobre tratar al cardenal Juan Francisco Albani... no conviene de ningún modo unirse a él, ni fiarse de su trato, que es doble y finge todo... Sabe Su Ema. cuán odioso es el nombre de los Albanis a la Francia, y que bastará para la desunión de sus cardenales de nuestro partido el saber, o sospechar, que SS.EE. tratan con dicho Juan Francisco...». ${ }^{55}$ El juicio de Azpuru acerca del cardenal Albani no iba mal encaminado; la doblez y el sinuoso comportamiento de Juan Francisco quedaron al descubierto cuando D'Aubeterre informó al embajador español de su pretendido acercamiento, al tiempo que lo intentaba con los españoles, del menor de los Albanis al cardenal francés Bernis. Llama la atención que Azpuru silenciara el encuentro tenido con los cardenales españoles: «Azpuru hace saber a Su Ema. [Solís] que habiendo estado esta mañana con el embajador de Francia, le ha participado que ayer tuvo un billete del cardenal de Bernis en el que le decía este Emmo. que Juan Francisco Albani solicitaba suu amistad y confianza y ser el único que en las presentes circunstancias podia proporcionar con su manejo una elección que fuese grata a las cortes. Nada le ha contestado Azpuru de que hacia igual solicitud con los Sres. españoles sin noticia de los franceses...». ${ }^{56} \mathrm{El}$ intencionado silencio de Azpuru avalaba las quejas que Bernis hizo a los cardenales españoles sobre sus tratos secretos con los Albanis. Azpuru, que sin duda había sopesado pros y contras de contar con el apoyo de los Albanis, veía en su apoyo la posibilidad de dar un giro definitivo a la marcha del cónclave. Por ello se mostró partidario de entablar negociaciones con los aborrecidos Albanis pero no de modo oficial: «si Juan Francisco Albani se insinuase por tercera persona a favor de Cavalchini podría V.Ema., por el mismo conducto, cultivar con su grande habilidad esta especie, y no despreciarla, sin que por ello se pudiesen dar por ofendidos los franceses, pues confiesa el mérito de dicho Cavalchini y aparentan tenerle inclinación.» ${ }^{57}$ Faltando sólo un día para la elección, Solís se lamentaba de las acusaciones, según él injustas e infundadas, que hacía Bernis a los españoles. El cardenal francés parecía haber descubierto el supuesto trato de Solís con el cardenal Albani y le acusaba de atentar contra los intereses de la liga borbónica.

El embajador español no pudo desmentir lo dicho por Bernis; aún así no contó a Grimaldi toda la verdad. En la carta dirigida a su superior, fechada el 18 de mayo, explicaba las acusaciones de Bernis y se rasgaba las vestiduras al comprobar cómo el cardenal francés daba más crédito a Juan Francisco que a los españoles; pero ocultaba que él mismo, seis días antes, autorizaba a Solis para que sondease a los Albanis «por tercera persona $\gg .{ }^{58}$

El incidente entre Bernis y los cardenales españoles vino a entorpecer, de nuevo, el complicado curso de las negociaciones dentro del cónclave. Las diferencias dentro

55. A.G.S., Estado, leg. 5013, Azpuru a Grimaldi, Roma, 10 de mayo de 1769.

56. A.G.S., Estado, leg. 5013, Azpuru a Solís, Real palacio de España, 2 de mayo de 1769.

57. A.G.S., Estado, leg. 5013, Azpuru a Solís, Real palacio de España, 12 de mayo de 1769.

58. A.G.S., Estado, leg. 5013, Azpuru a Solís, Real palacio de España, 12 de mayo de 1769. 
de la liga borbónica se agrandaban por momentos. Vista la situación de punto muerto y los reiterados enfrentamientos que se daban en el seno del Sacro colegio, Azpuru decidió sondear la alternativa planteada por los Albanis permitiendo, como hemos visto, el acercamiento de sus cardenales al menor de los Albanis. Agotadas las posibilidades reales de Sersale, Cavalchini y Stoppani, el embajador español comenzó a apostar, con fuerza, por un purpurado que hasta el mes de mayo había pasado inadvertido: fray Lorenzo Ganganelli. Monseñor Azpuru decidió retirar el apoyo de España a Cavalchini y concentrarlo en el único fraile del Sacro colegio; contando con el respaldo del partido de los Albanis y el de los coronas el cardenal Ganganelli estaría muy cerca de acceder al solio de san Pedro. Pero estas negociaciones se llevaron a espaldas de los franceses, generando el consiguiente malestar: «Ha venido esta noche el embajador de Francia a este palacio y ha dicho que acababa de recibir un billete del cardenal de Bernis, en el que le hacía la pregunta si podría ir por Ganganelli, pues se trataba de proponerlo mañana, y que los señores cardenales españoles nada le habian dicho del tratado, que habían tenido con el cardenal Juan Francisco Albani, por quien Su Ema. lo había sabido, y como extrañando el embajador que se hubiese dado paso alguno sin inteligencia de sus cardenales, ha manifestado que le disgustaba dicha propuesta, porque era intempestiva, y sólo serviría para arruinar a dicho Ganganelli, de modo que no pudiese volver a juntar partido...»..$^{59}$

A pesar de la tensión generada dentro de la liga borbónica, la figura de Ganganelli estaba siendo considerada seriamente por todos los partidos del cónclave. La caída de todos los candidatos propuestos hasta el momento, los cuatro meses de encierro en las estancias vaticanas y el peligro de una nueva situación de bloqueo, afianzaron sus posibilidades.

Al poco de iniciarse el cónclave, y con motivo de las entrevistas mantenidas por los embajadores borbónicos con los cardenales que componían el colegio cardenalicio, Azpuru celebró un largo encuentro con fray Lorenzo, y ello levantó ciertas suspicacias. Azara lo comentaba a Roda: «El pueblo hace tres días que ha hecho papa a Ganganelli con motivo de que Azpuru hace otros tantos días que le hizo una visita de cuatro horas y media. ¿Quién habrá engañado a quién? Este proyecto conviene a Azpuru, pero mienten todas mis ideas si el tal fraile llega a coronarse». ${ }^{60}$ Ganganelli se encontraba aún lejos de la máxima dignidad de la Iglesia, y Azara pensaba que ni siquiera llegaría a reunir la fuerza suficiente para ser propuesto al colegio cardenalicio: «...tardaré más en creer que este sujeto llegue nunca al candelero, aún cuando fuese verdad todo lo que de la protección de España se abulta por aquí. La verdad pura, sin abultar fachendas, es que sin un milagro ninguno puede ser papa en el punto en que se está, porque no hay unida materia para amasarlo». ${ }^{61}$ Un mes más tarde el tono de las cartas de Azara apuntaba en otra dirección. Fray Lorenzo recababa más

59. A.G.S., Estado, leg. 5013, Azpuru a Solís, Real palacio de España, 16 de mayo de 1769.

60. Azara a Roda, Roma, 16 de febrero de 1769, El espíritu..., t. I, p. 222.

61. Azara a Roda, Roma, 6 de abril de 1769, El espíritu..., t. I, pps. 250-251. 
apoyos del colegio y su candidatura iba ganando fuerza, al tiempo que la de otros purpurados iba menguando. El agente de preces recordaba, irónicamente, sus prevenciones hacia Ganganelli: «De jesuitas y su extinción, es ya punto sentado no hablar. Si sale papa Ganganelli, la ejecutará, el que siendo fraile, dedicaba sus conclusiones al Padre Retz, general entonces solipsorum; y que de cardenal, hizo lo que sabemos con Mesengui. En fin vd. discurra lo que ha de salir de toda esta pepitoria». ${ }^{.2} \mathrm{El}$ día 12 de mayo Azpuru se hacía eco de un rumor por el que presuntamente Ganganelli renunciaba a ser propuesto al Sacro colegio, y solicitaba noticias al conclavista de Solís, Sebastián García, para confirmar o desmentir dicha información. ${ }^{63}$ Desmentida la renuncia de Ganganelli al papado, Azpuru comenzó a trabajar seriamente para sacar adelante su candidatura.

El cardenal arzobispo de Sevilla advertía a Azpuru que los cardenales franceses, a día 14 de mayo, y a pesar de las nuevas órdenes venidas de Francia, no se daban por enterados de que había que promover a Ganganelli. El cardenal español no veía muchas posibilidades en la candidatura del franciscano: «Juzga el cardenal de Solís será muy dificultoso que ascienda al papado este Emo....». ${ }^{64}$ Sin embargo, pocas horas después Solis se declaraba firme partidario de apoyar a Ganganelli y proponer su candidatura al Colegio. ${ }^{65}$ Resulta llamativo el repentino cambio de pensar del purpurado español sobre fray Lorenzo. Tan sólo dos días antes, el 14 de mayo, veía «muy dificultoso» que Ganganelli obtuviera la mitra papal; además Solís se decantaba abiertamente hacia el franciscano, «con el que tiene anterior amistad y práctica de su carácter». ¿QQué motivo o motivos existían para que en tan reducido espacio de tiempo las posibilidades de Ganganelli se vieran tan acrecentadas? El apoyo de los Albanis, había sido un factor determinante. Las reiteradas acusaciones de Bernis a los españoles por sus conversaciones secretas con Juan Francisco, desmentidas de forma poco convincente por los implicados, corroboraban el afianzamiento de Ganganelli, y el incremento de sus apoyos en el colegio cardenalicio. Por otra parte, resulta difícilmente creíble que ni Azpuru, ni Solís, después de haber mantenido encuentros con Ganganelli no sondearan, cuando no lo hicieran abiertamente, cuál era su actitud ante la Compañía de Jesús.

El 17 de mayo Azpuru escribía a Solís el siguiente planteamiento: «llevando por norte la unión encargada por nuestra corte con los cardenales franceses, aunque juzga [Azpuru] como S.Ema. [Solis] que el cardenal Ganganelli es más a propósito que Stoppani para ocupar la silla de San Pedro... podrán SS.EE. proponer a los cardenales franceses que si quisieren que siga su curso la propuesta de dicho Stoppani, suspendida a su instancia, están prontos SS.EE., como siempre lo han estado, a unirse a ellos, y votar por el mencionado Stoppani; pero si insistiesen en dicha suspensión

62. Azara a Roda, Roma, 11 de mayo de 1769, El espíritu..., t. I, p. 277

63. A.G.S., Estado, leg. 5013, Azpuru a Sebastián Garcia, Real palacio de España, 12 de mayo de 1769.

64. A.G.S., Estado, leg. 5013, Solís a Azpuru, Del cónclave, 14 de mayo de 1769.

65. A.G.S., Estado, leg. 5013, Solís a Azpuru, Del cónclave, 16 de mayo de 1769. 
por ahora, dejar que corra la propuesta de Ganganelli...». ${ }^{66}$ Bien conocía Azpuru la respuesta de los cardenales galos a su planteamiento. Stoppani, candidato de los franceses, se había «quemado» sin haber sido, siquiera, propuesto al colegio cardenalicio. Mientras el embajador español trataba de calmar los ánimos de los franceses, Orsini le enviaba un billete «donde manifestaba su deseo de servirle» y exponía cómo se encontraba la propuesta de Ganganelli. ${ }^{67}$ Según Azara el embajador español y el cardenal Ganganelli se afanaban, esa misma jornada, para culminar con éxito sus proyectos: «Hoy el que más enreda es el fraile Ganganelli. El movimiento que se da aturde aún a los mismos que antes creían conocerle. Lo que le da alas para esto es saber que Azpuru trabaja bajo mano por él, fuera y dentro...». ${ }^{68} \mathrm{El}$ mismo día que Orsini escribía a Azpuru, el cardenal Solís anunciaba en una breve misiva el triunfo de la candidatura de Ganganelli: «ahora que son las diez de la noche tengo el gusto de participarle a V.Ilma. que mañana en el primer escrutinio o a más tardar en el acceso tendremos papa al cardenal Ganganelli... Tenemos asegurados los 18 votos nuestro partido, y según ha expuesto el cardenal Rezzonico a... Bernis y Cerda habrá 20 votos de los suyos, por lo que contamos 38... Tengo la satisfacción de que hemos convenido todos unánimes...». ${ }^{69}$

En efecto, en el escrutinio número 185 y último, el cardenal Ganganelli fue elegido unánimemente. Un testimonio excepcional, el diario del cardenal Pirelli, asistente al cónclave, recogía el resultado de la votación: Ganganelli 45 votos, Rezzonico 1 voto.$^{70} \mathrm{El}$ voto obtenido por Rezzonico se debía más que a un factor testimonial a una fórmula de cortesía: «Nel pronunziarsi i voti, quando Calino, ultimo scrutatore, arrivó al 31 , si alzó, si cavò il berettino, gli fece reverenza e lo pronunziò con voce più alta moderatamente del solito. Finito lo scrutinio, si trovaron tutti $i$ voti per l'eletto, a riserva del suo, che fu dato da lui a Rezzonico»."

Después de haber superado muchas dificultades, y descartados los candidatos óptimos para los borbones, Ganganelli se convirtió en una figura de consenso de los principales partidos del cónclave. Las conversaciones secretas, que habían trascendido a pesar de Azpuru, de Juan Francisco Albani con los españoles tuvieron como eje central la candidatura de fray Lorenzo. Pese al enfado y desconcierto inicial de Bernis, la figura de Ganganelli estaba siendo estudiada por todas las facciones del cónclave: «Conoció el partido Rezzonico, que al regio se le iban uniendo muchas voces, y por consiguiente, que se exponía a una notoria contrariedad, se acordó por dicho partido el de seguir el de las tres cortes». ${ }^{72}$

66. A.G.S., Estado, leg. 5013, Azpuru a Solís, Real palacio de España, 17 de mayo de 1769.

67. A.G.S., Estado, leg. 5013, Orsini a Azpuru, Dal cónclave, 18 de mayo de 1769.

68. Azara a Roda, Roma, 18 de mayo de 1769, El espíritu..., t. I, 18 de mayo de 1769.

69. A.G.S., Estado, leg. 5013, Solís a Azpuru, Del cónclave, 18 de mayo de 1769.

70. BERRA, L.: «Il diario del conclave di Clemente XIV del cardinale Filippo Maria Pirelli» en Archivio della Societá di Storia Patria, 16-17, (1962-63), Roma, p. 315.

71. Ibídem, p. 316.

72. A.G.S., Estado, leg. 5013, Solís a Grimaldi, Roma, 25 de mayo de 1769. 
Rezzonico se comprometió a participar a sus cardenales afectos la propuesta de Ganganelli, y solicitó tiempo "para que lo premeditasen con la mayor madurez». ${ }^{73}$ Tras una tensa espera de día y medio, Solís explicaba a Grimaldi la rapidez con que se precipitaron los acontecimientos: «Ganganelli fue hecho papa en doce horas». ${ }^{74}$

Las reacciones de Madrid y de sus representantes en Roma ante la elección de Ganganelli no se hicieron esperar. Azpuru se deshacía en elogios ${ }^{75}$; el cardenal De la Cerda escribía a Grimaldi una carta felicitándose calurosamente por tan acertada elección ${ }^{76}$; el cardenal de Solís, dejando a un lado los parabienes y enhorabuenas, iba más allá, hablando de la próxima extinción de la Compañía ${ }^{\eta}$.

A los diez días de la elección la noticia fue conocida en Madrid, destacó, entre todas, la efusiva acogida que le dispensó el confesor de Carlos III. ${ }^{78}$ Grimaldi, más comedido, comunicaba a Azpuru la buena impresión que había causado a Carlos III la noticia. El Secretario de Estado acogía la exaltación de Ganganelli al solio pontificio con cierta reserva: «S.M. ha celebrado infinito la elección por ser uno de los pocos de quien se esperaba atendiesen a sus justas solicitudes y procurase la buena armonía entre la curia romana y las cortes católicas. Ahora nos falta experimentarlo». ${ }^{7 \%}$

A principios de junio de 1769 , cinco meses después de haber sido planteada al desaparecido Clemente XIII, Azpuru volvía a exponer al Sumo pontífice recién elegido la necesidad de extinguir a la Compañía de Jesús. Cerrado ya el intervalo que había supuesto el cónclave, las cortes católicas debían retomar sus solicitudes formales ante el Vaticano para zanjar, definitivamente, el asunto jesuítico. Tras un primer encuentro de cortesía, celebrado el 25 de mayo, el embajador español aprovechó su segunda audiencia con Clemente XIV, que tuvo lugar el 3 de junio, para tratar de la espinosa cuestión jesuítica: «le toqué el punto de la extinción de los jesuitas y dije a $\mathrm{Su}$ Beatitud que ese era el asunto que miraba S.M. con la mayor atención... y me respondió que bien sabía yo cómo pensaba, que le diese un poco de tiempo, y no dudase que contentaría a S.M. como lo vería. Díjele que así lo esperaba y tenía por seguro, que corresponderían sus operaciones en el pontificado al sabio y recto modo de pensar que me había manifestado varias veces cuando era cardenal...». ${ }^{80}$

Mucho debió sentir Azpuru el relevo «voluntario» de su colega D'Aubeterre y su sustitución por Bernis. D'Aubeterre en todo momento se había mostrado partidario de presionar al Vaticano para terminar definitivamente con la Compañía. Por el con-

73. A.G.S., Estado, leg. 5013, Extracto de lo acaecido en el día 17 y 18 del mes pasado por lo tocante a la elección de Clemente XIV, cardenal de Solis, Roma, 28 de junio de 1769.

74. A.G.S., Estado, leg. 5013, Solís a Grimaldi, Roma, 25 de mayo de 1769.

75. A.G.S., Estado, leg. 5013, Azpuru a Sebastián García, Real palacio de España, 19 de mayo de 1769.

76. A.G.S., Estado, leg. 5013, El cardenal De la Cerda a Grimaldi, Roma, 25 de mayo de 1769.

77. A.G.S., Estado, leg. 5013, Solís a Grimaldi, Roma, 25 de mayo de 1769.

78. A.G.S., Estado, leg. 5013, Joaquín de Osma a Solís, Aranjuez, 29 de mayo de 1769.

79. A.G.S., Estado, leg. 5013, Grimaldi a Azpuru, Aranjuez, 30 de mayo de 1769.

80. A.M.AA.EE., Santa Sede, leg. 332, Azpuru a Grimaldi, Roma, 8 de junio de 1769. 
trario, Bernis mientras estuvo en el cónclave no quiso participar en esas negociaciones y rehusó implicarse en las reivindicaciones de la corte de España y de su propio embajador. Azpuru trató de recomponer el «frente» constituido por los borbones ante el Vaticano a finales de 1768 y principios de 1769 para volver a exigir la supresión de la Orden. Conociendo el relevo de D'Aubeterre por Bernis acudió a este último «y le pregunté si había recibido las órdenes e instrucciones que esperaba el marqués D'Aubeterre para la instancia de la extinción de la Compañia, pues sabía que aquél no las habia recibido, y me dijo Su Emma. que, aunque todavía no habían venido, le escribia el Sr. duque de Choiseul que se debería proponer ante todas dicha extinción... Díjele entonces lo que el papa me había asegurado en la audiencia de que dí cuenta a V.E. y se explicó que tenía la mayor seguridad y nunca había dudado, que Su Santidad hará dicha extinción, y añadió que se lo había ofrecido dos días antes de. su elección en papa, pero que era preciso darle tiempo para que no compareciera dicha providencia intempestiva, ni forzada». ${ }^{81}$ Era realmente cierto lo dicho por Bernis a Azpuru? Resulta extraño el supuesto ofrecimiento de Ganganelli a Bernis, sobre todo si recordamos que el cardenal galo jamás quiso plantear a ningún cardenal asunto alguno relacionado con la extinción de la Compañía.

Finalmente el gobierno galo autorizaba a su nuevo ministro en Roma para que se retomasen las reclamaciones ya hechas al papa Rezzonico.

Magros resultados se obtuvieron de las nuevas negociaciones con el sucesor de Clemente XIII. No fue sino tres años después cuando se lograron encaminar las negociaciones que condujeron a la supresión del Instituto fundado por San Ignacio.

La elección de Clemente XIV siempre estuvo ensombrecida por la sospecha de un posible pacto simoníaco. En la documentación analizada no aparece ninguna referencia a un pacto o documento formal firmado por fray Lorenzo. No obstante, sí hay suficientes indicios que corroboran la idea de un cardenal Ganganelli ambiguo, como pocos, en su comportamiento. En los prolegómenos del cónclave el embajador español celebró una extensa entrevista con dicho cardenal. Probablemente el franciscano habló en términos equívocos y Azpuru modificó su criterio acerca de él. Faltando sólo dos jornadas para la elección, Azpuru escribía al conclavista de Solís, Sebastián García, dando a entender que conocía el modo de pensar de Ganganelli, e insinuaba, al tiempo, que sabía algo más: «Me consta cuál es el [modo de pensar] de Ganganelli, su particular trascendencia y amor que tiene a nuestro rey... y por lo mismo no extraño lo que a Vm. [Sebastián García] le ha confiado el cardenal consabido, y retendré en mí con el mayor secreto, aunque sin darme por entendido de la especie comunicada haré por confortar a este embajador [D'Aubeterre], que en su corazón siempre ha querido a Ganganelli, y alguna vez se ha explicado conmigo deseando que se eligiese papa» ${ }^{82}$ ¿Conocía realmente algo que implicara a Ganganelli?, ¿qué era lo «que retenía con el mayor secreto»?, y, ¿por qué no hizo mención alguna en su correspondencia con Grimaldi de «esa especie comunicada»?

81. A.M.AA.EE, Santa Sede, leg. 332, Azpuru a Grimaldi, Roma, 15 de junio de 1769.

82. A.G.S., Estado, leg. 5013, Azpuru a Sebastián García, Real palacio de España, 16 de mayo de 1769 
Después de la elección, Azpuru escribía a Grimaldi «que la extinción de los jesuitas no se pudo asegurar en el cónclave» ${ }^{83}$, pero no dudaba de que bajo el nuevo pontificado los jesuitas tenían sus días contados: «[Ganganelli] se explicó en términos que el cardenal Solís no dudó que haría dicha extinción si ascendiese al pontificado... tenemos argumentos no equivocados de que condescenderá... el otro argumento es haberme dicho hablando [Ganganelli], aunque no claramente, del asunto de la extinción que se quitarian las espinas... ${ }^{84}$.

Solís, en un escrito con fecha de 28 de junio de 1769 , hablaba de «tratos secretos» mantenidos con Ganganelli y por ello se decantaba hacia este purpurado: «Procuró disuadir el cardenal de Solís a dichos cardenales [Bernis, Luynes y Orsini]... asegurándoles por el anterior trato que tenía secretamente con el cardenal Ganganelli, que ninguno sería más a propósito para ocupar la silla de San Pedro.» ${ }^{85}$

Resulta llamativo que el único cardenal regular, con su sinuoso carácter fuera capaz de confundir a todas las facciones del cónclave y conseguir sus apoyos para elevarlo a la máxima dignidad de la Iglesia. Insistiendo en lo ya aludido anteriormente, la documentación manejada no aporta ninguna prueba formal que haga referencia a un compromiso de Ganganelli obligándose a extinguir a la Compañía de Jesús. Lo que sí pudo asegurar Azpuru a Grimaldi fue que «el papa antes de serlo no hizo la promesa, ni se negó a ella.» ${ }^{86}$

83. A.M.AA.EE., Santa Sede, leg. 332, Azpuru a Grimaldi, Roma, 25 de mayo de 1769.

84. Ibídem.

85. A.G.S., Estado, leg. 5013, Extracto de lo acaecido en el día 17 y 18 del mes pasado por lo tocante a la elección de Clemente XIV, cardenal de Solís, Roma, 28 de junio de 1769.

86. A.M.AA.EE., Santa Sede, leg. 5013, Azpuru a Grimaldi, Roma, 25 de mayo de 1769. 